\title{
RETINOPATÍA DEL PREMATURO EN LA REGIÓN DE MURCIA (ESPAÑA). INCIDENCIA Y GRAVEDAD
}

\section{RETINOPATHY OF PREMATURITY IN THE MURCIA REGION OF (SPAIN). INCIDENCE AND SEVERITY}

\author{
HERNÁNDEZ M ${ }^{1}$, ORDUNA C ${ }^{2}$, BOSCH V ${ }^{3}$, SALINAS R $^{4}$, ALCARAZ JL ${ }^{4}$, MARÍN JM ${ }^{1}$
}

\section{RESUMEN}

Objetivo: Conocer la incidencia actual y gravedad de la Retinopatía del Prematuro (ROP) en la región de Murcia.

Método: Estudio retrospectivo de todos los prematuros nacidos en la región de Murcia durante 2004 y que fueron sometidos a cribado ROP. Los criterios de cribado fueron: Recién nacidos pretérmino (RNPT) con $\leq 32$ semanas de edad gestacional (EG) o RNPT con $\leq 1.500 \mathrm{~g}$ de peso al nacer $(\mathrm{PN})$. Se registró sexo, EG, PN, paridad y evaluación oftalmológica. Se realizó un estudio descriptivo y analítico.

Resultados: La muestra la forman 115 prematuros con una EG media de 29,67 semanas y un PN medio de $1.325 \mathrm{~g}$. La incidencia global de ROP fue del $32,1 \%$, y de ROP grave del $15,6 \%$ del total de la muestra. El 88,8\% de los prematuros con ROP grave tenía $\leq 29$ semanas EG o $\leq 1000$ g PN. Estadísticamente existían diferencias significativas, $(p<$ 0,001 ) para la EG y el PN, entre los prematuros con ROP y sin ROP.

Conclusiones: Un tercio de los prematuros cribados para ROP en la región de Murcia desarrolla la
ABSTRACT

Objective: To determine the incidence and severity of Retinopathy of Prematurity (ROP) in the Murcia region of Spain.

Methods: This was a retrospective study of all infants born preterm in the Murcia region during 2004, who were screened for ROP. Our screening criteria were: Infants with a gestational age $\leq 32$ weeks (GA) or with a birth weight $\leq 1500 \mathrm{~g}$ (BW). We evaluated sex, GA, BW, parity and ophthalmologic evaluation. We then performed a descriptive study and a statistical analysis.

Results: The study sample involved 115 infants with an average GA of 29.67 weeks and BW of $1325 \mathrm{~g}$. The incidence of ROP and severe ROP was $32.1 \%$ and $15.6 \%$ respectively. The likelihood of the premature infant developing severe ROP was found to be $88.8 \%$ if it was born before 29 weeks GA or weighed less than $1000 \mathrm{~g}$ BW. Significant differences $(p<0.001)$, in terms of GA and BW between infants with and without ROP, were found. Conclusions: A third of the preterm infants screened for ROP in the Murcia region developed the disease, and the $50 \%$ of these required treatment.

\footnotetext{
Recibido: 10/1/07. Aceptado: 4/6/08.

Hospital Universitario Virgen de la Arrixaca. Murcia. España.

${ }^{1}$ Licenciado en Medicina. Servicio de Oftalmología.

2 Doctor en Medicina. Servicio de Oftalmología.

3 Doctor en Medicina. Servicio de Neonatología.

${ }^{4}$ Licenciado en Medicina. Servicio de Neonatología.

Correspondencia:

Mercedes Hernández Martínez

Servicio de Oftalmología

Hospital Universitario Virgen de la Arrixaca

Ctra. Madrid-Cartagena, km. 7

30120 El Palmar (Murcia)

España

E-mail: mercehernandez@terra.es
} 
enfermedad y el $50 \%$ de ellos precisa tratamiento. Los resultados también indican que los criterios de cribado actuales son adecuados para nuestra población. Con criterios más restrictivos no se detectarían todos los pacientes con ROP grave. Para modificar el protocolo de cribado ROP precisamos más estudios.

Palabras clave: Retinopatía del prematuro, incidencia, cribado.
The current screening criteria used in our region are appropriate. If more restrictive criteria were used, some severe ROP cases would not be detected. In order to be able to modify the current guidelines for screening for ROP, further studies are required (Arch Soc Esp Oftalmol 2008; 83: 423-428).

Key words: Retinopathy of Prematurity, incidence, screening.

\section{INTRODUCCIÓN}

La incidencia de la Retinopatía del Prematuro (ROP) se asocia con las tasas de supervivencia y con la gravedad del proceso sistémico en el recién nacido pretérmino (RNPT) y se ve influida por estos factores (1). El grupo CRYO-ROP encontró una incidencia global de ROP del 65,8\% (2) y el grupo ET-ROP, cuyo estudio se realiza 15 años más tarde, ya en pleno siglo XXI, encuentra una incidencia similar, $68 \%$, pero obtiene mayor incidencia de ROP grave (3). Los recientes avances en neonatología han influido sobre la distribución de la ROP. La incidencia global permanece estable pero hay un aumento de ROP grave entre los más inmaduros y una disminución entre los más maduros (4). También se ha demostrado, a finales de la última década, un aumento de ROP grave entre los prematuros con muy bajo peso al nacer, que parece ser independiente del incremento en la supervivencia (5). Ciertos tratamientos practicados en los muy inmaduros, necesarios para asegurar la supervivencia, podrían aumentar el riesgo de ROP grave (6).

En la región de Murcia se desconoce la incidencia de la ROP. Con este estudio se pretende conocer la situación actual en cuanto a incidencia y gravedad de la ROP en nuestra población.

\section{SUJETOS, MATERIAL Y MÉTODO}

Han sido incluidos todos los prematuros nacidos entre el 1 de enero y el 31 de diciembre de 2004 en la región de Murcia, que ingresaron en la Unidad de Cuidados Intensivos Neonatales (UCIN) o en la unidad de cuidados intermedios neonatales del Hospital Universitario Virgen de la Arrixaca (HUVA), que sobrevivieron más de 28 días y que fueron sometidos a cribado ROP. Los criterios de inclusión en el cribado ROP eran: RNPT con $\leq 32$ semanas de edad gestacional (EG), RNPT con $\leq 1.500 \mathrm{~g}$ de peso al nacer (PN) y RNPT fuera de estos parámetros, con curso inestable, a criterio del pediatra. Este protocolo de cribado está basado en las recomendaciones de las Academias Americanas de Pediatría y Oftalmología (7), modificado para nuestra Unidad. El examen de fondo de ojo se realizó bajo midriasis farmacológica con colirios de fenilefrina al 2,5\% y ciclopentolato al 0,5\%. De rutina se utilizó el oftalmoscopio binocular indirecto y lupa de 28D. En casos de blefarofimosis o no visualización de la unión entre retina vascular y avascular, se utilizó el blefarostato infantil e indentación escleral bajo anestesia tópica. El primer examen oftalmológico se realizó entre la $4 .^{\mathrm{a}}$ y la $6 .^{\mathrm{a}}$ semana de vida, nunca antes de la 31 semana de edad postmenstrual, con revisiones cada 1-2 semanas hasta la vascularización retiniana completa (8). Cada niño fue calificado con el grado máximo de retinopatía detectado según la Clasificación Internacional de la ROP (ICROP) $(9,10)$. El tratamiento con láser diodo se indicó en los pacientes con retinopatía aguda grado 3 de la ICROP (9), que alcanzaron la calificación de enfermedad UMBRAL, según definición de CRYO-ROP (11), o enfermedad PREUMBRAL según definición de ET-ROP (12). El tratamiento quirúrgico se indicó en los pacientes con retinopatía grado 4 ó 5 de la ICROP (10). En todos los casos con necesidad de tratamiento se obtuvo un consentimiento informado de los padres o tutores.

Para este estudio se han definido tres grupos: Grupo A (NO ROP); Grupo B (ROP LEVE), incluye los pacientes con retinopatía que no precisaron tratamiento; Grupo C (ROP GRAVE), incluye los pacientes con retinopatía que precisaron laserterapia o cirugía vitreorretiniana. Se registró sexo, EG, PN, paridad y evaluación oftalmológica. Para el análisis estadístico de los resultados se utilizó el programa 
SPSS para Windows versión V12.0.1 (SPSS for Windows, SPSS Inc., Chicago, USA). La estadística descriptiva se realizó mediante medidas de centralización (media) y de dispersión (desviación típica). Para el análisis de comparación de varias medias (EG y PN) entre los tres grupos se utilizó el test de ANOVA de un factor, y la prueba de Scheffé para verificar entre qué grupos existían diferencias.

\section{RESULTADOS}

La muestra del estudio la componen 115 prematuros de los cuales 100 tenían $\leq 32$ semanas EG, 86 tenían $\leq 1.500$ g PN y 10 tenían $>32$ semanas EG y $>1.500$ g PN. La incidencia global de ROP es del $32,1 \%$ y de ROP grave del $15,6 \%$. Los prematuros cribados con $>32$ semanas EG y $>1.500 \mathrm{~g}$ PN no desarrollaron ROP. De los 115, 75 son hombres $(65,2 \%)$ y 40 mujeres (34,8\%). De los 115 RNPT,
15 proceden de paridad múltiple (13\%). La EG media de la muestra es de 29,67 semanas con una desviación típica (DS) de 2,3 y un rango de 25-35. El PN medio de la muestra es de $1.325 \mathrm{~g}$ con una DS de 365 y un rango de 560-2.850. La tabla I muestra las características de cada uno de los pacientes con ROP grave. La tabla II recoge la EG media y el PN medio por grupos.

Se diagnosticó ROP grave en un paciente con 32 semanas EG y 1.305 g PN y en otro con 31 semanas EG y $1.400 \mathrm{~g}$ PN. El resto de prematuros del grupo $\mathrm{C}$ tenían $\leq 29$ semanas $\mathrm{EG} \mathrm{o} \leq 1.000 \mathrm{~g} \mathrm{PN}$. Las tablas III y IV recogen la distribución de la muestra entre los grupos A, B y C, según intervalos de EG y PN respectivamente.

La comparación de EG y PN entre los grupos A, B y C demostró diferencias estadísticamente significativas entre grupos, para $\mathrm{EG}(\mathrm{F}=23,771$; $\mathrm{p}<$ $0,001)$ y para $\mathrm{PN}(\mathrm{F}=12,727$; $<<0,001)$. Con la prueba de Scheffé se comprobó que esta diferencia

Tabla I. Características de los pacientes con ROP grave

\begin{tabular}{rlcccc}
\hline Paciente N. & Sexo & $\begin{array}{c}\text { Edad gestacional } \\
\text { (semanas) }\end{array}$ & $\begin{array}{c}\text { Peso al nacer } \\
\text { (gramos) }\end{array}$ & $\begin{array}{c}\text { Grado retinopatía } \\
\text { alcanzado }\end{array}$ & Evolución \\
\hline 1 & Mujer & 29 & 1.200 & 3 & Favorable \\
2 & Hombre & 25 & 770 & 3 & Favorable \\
3 & Hombre & 25 & 700 & 3 & Favorable \\
4 & Mujer & 31 & 1.400 & 3 & Favorable \\
5 & Hombre & 28 & 1.400 & 3 & Favorable \\
6 & Hombre & 27 & 870 & 3 & Favorable \\
7 & Hombre & 25 & 960 & 4 & Favorable \\
8 & Hombre & 26 & 720 & 3 & Favorable \\
9 & Hombre & 27 & 1.300 & 3 & Favorable \\
10 & Hombre & 25 & 960 & 3 & Favorable \\
11 & Mujer & 25 & 1.000 & 3 & Favorable \\
12 & Hombre & 27 & 970 & 3 & Favorable \\
13 & Hombre & 26 & 780 & 3 & Favorable \\
14 & Hombre & 27 & 1.100 & 3 & Favorable \\
15 & Mujer & 28 & 1.170 & 5 & Desfavorable \\
16 & Mujer & 32 & 1.305 & 3 & Favorable \\
17 & Hombre & 29 & 830 & 3 & Favorable \\
18 & Hombre & 28 & & 300 & 3 \\
\hline \hline
\end{tabular}

Tabla II. Edad gestacional media y peso medio al nacer de los grupos A, B y C

\begin{tabular}{lcccccc}
\hline Grupos & Media & $\begin{array}{c}\text { Edad gestacional } \\
\text { Desviación típica }\end{array}$ & Rango & Media & $\begin{array}{c}\text { Peso al nacer } \\
\text { Desviación típica }\end{array}$ & Rango \\
\hline $\begin{array}{l}\text { Grupo A (NO ROP) } \\
\mathrm{n}=78\end{array}$ & 30,5 & 1,9 & $26-35$ & 1.426 & 365 & $560-2.850$ \\
$\begin{array}{l}\text { Grupo B (ROP LEVE) } \\
\mathrm{n}=19\end{array}$ & 28,7 & 1,6 & $25-32$ & 1.187 & 215 & $770-1.540$ \\
$\begin{array}{l}\text { Grupo C (ROP GRAVE) } \\
\mathrm{n}=18\end{array}$ & 27,2 & 2,1 & $25-32$ & 1.029 & 229 & $700-1.400$ \\
\hline \hline
\end{tabular}


Tabla III. Distribución de la muestra entre los grupos A, B y C por intervalos de edad gestacional

\begin{tabular}{lccc}
\hline Intervalo edad gestacional & Grupo A (NO ROP) & Grupo B (ROP LEVE) & Grupo C (ROP GRAVE) \\
\hline $25-26(\mathrm{n}=10)$ & $2(20 \%)$ & $1(10 \%)$ & $7(70 \%)$ \\
$27-29(\mathrm{n}=39)$ & $18(46,2 \%)$ & $12(30,8 \%)$ & $9(23,1 \%)$ \\
$30-32(\mathrm{n}=51)$ & $43(84,3 \%)$ & $6(11,8 \%)$ & 0 \\
$>32(\mathrm{n}=15)$ & $15(100 \%)$ & 19 & 18 \\
TOTAL $\mathrm{n}=115$ & 78 & & $18 \%)$ \\
\hline \hline
\end{tabular}

Tabla IV. Distribución de la muestra entre los grupos A, B y C por intervalos de peso al nacer

\begin{tabular}{lccc}
\hline Intervalo peso al nacer & Grupo A (NO ROP) & Grupo B (ROP LEVE) & Grupo C (ROP GRAVE) \\
\hline $500-1.000(\mathrm{n}=21)$ & $7(33,3 \%)$ & $4(19 \%)$ & $10(47,6 \%)$ \\
$1.001-1.250(\mathrm{n}=29)$ & $18(62,1 \%)$ & $7(24,1 \%)$ & $4(13,8 \%)$ \\
$1.251-1.500(\mathrm{n}=36)$ & $26(72,2 \%)$ & $6(16,7 \%)$ & $4(11,1 \%)$ \\
$>1.501(\mathrm{n}=29)$ & $27(93,1 \%)$ & $2(6,9 \%)$ & 0 \\
TOTAL $\mathrm{n}=115$ & 78 & 19 & 18 \\
\hline \hline
\end{tabular}

existía entre el grupo A con los grupos B y C (tanto para EG como para $\mathrm{PN}$ ), pero no existía entre el grupo $\mathrm{B}$ y el $\mathrm{C}$.

\section{DISCUSIÓN}

Este es el primer estudio que aporta datos de incidencia de ROP en la región de Murcia. El HUVA es el único centro de la región que dispone de UCIN, por lo que la gran mayoría de RNPT con $\leq 1.500 \mathrm{~g}$ PN ingresa en él. En el año 2004 nacieron 132 prematuros con $\leq 1.500 \mathrm{~g}$, en Murcia y provincia, 101 de los cuales sobrevivieron más de 28 días. La muestra estudiada por nosotros tiene 86 prematuros con $\leq 1.500 \mathrm{~g}$ PN, que supone el $85,1 \%$ de los supervivientes con estas características, por lo que consideramos la muestra representativa de la región.

La incidencia global de ROP, hallada con el método descrito, ha sido del $32,1 \%$ y la incidencia de ROP grave del 15,6\% del total de la muestra. La incidencia de la ROP y su curso a través del tiempo han sido y siguen siendo objeto de debate. La comparación entre los diferentes estudios publicados tiene un valor limitado porque pueden tener distintos criterios de cribado o ser muestras muy pequeñas (3). Con criterios de cribado comunes, $1.500 \mathrm{~g}$ $\mathrm{PN}$, nuestros resultados de incidencia global de ROP son similares a los publicados por Larsson 36,4\% (4), Montañez 33,1\% (13), Grunauer 31\% (14), y Martín 29,2\% (15). En cambio, estamos por debajo de los grupos con criterios de 1.250 g PN:
CRYO-ROP 65,8\% (2), ET-ROP 68\% (3), y Pallás $50 \%$ (16). Al comparar la incidencia de ROP grave de nuestro estudio con otras series, observamos que es ligeramente superior a la publicada por Larsson $12,3 \%$ (4) o Montañez 12,7\% (13). Sin embargo, es muy superior a la de Grunauer 5\% (14), Martín $5,6 \%$ (15) o Pallás 6,1\% (16). Esta diferencia podría explicarse en parte por los criterios de indicación de tratamiento considerados en cada estudio. En nuestra serie se aplicó laserterapia en fase preumbral a cinco pacientes, con extensión inferior a umbral de retinopatía grado 3 en zona I (paciente n. ${ }^{\circ} 2$ ), o en zona II (pacientes n. ${ }^{\circ} 3,10,15$ y 18) y enfermedad plus asociada. La indicación de tratamiento precoz en estos niños se basó en las recomendaciones del grupo ET-ROP del año 2003 (12).

Aunque el $88,8 \%$ de niños con ROP grave de nuestra serie tienen $\leq 29$ semanas EG ó $\leq 1.000 \mathrm{~g}$ $\mathrm{PN}$, con criterios más restrictivos, se habrían omitido dos casos de ROP grave, que tenían 32 semanas EG y 1.305 g PN y 31 semanas EG y 1.400 g PN, respectivamente. Los programas de cribado se elaboran básicamente sobre 1 ó 2 criterios, EG y/o PN, y su valor generalmente es consensuado entre oftalmólogos y pediatras del mismo centro. Para comparar las características de los prematuros que desarrollaron ROP grave entre países con distinto nivel de desarrollo, se realizó una encuesta a oftalmólogos entre los años 1996-2002, donde se recogía EG y PN de los protocolos de cribado, así como los resultados y características de los prematuros que recibieron tratamiento. Los países se dividían en 
tres categorías: alto, moderado y bajo nivel de desarrollo. España no aparece referenciada. Los resultados mostraron que niños más maduros y con más peso están desarrollando ROP grave en países con bajo y moderado nivel de desarrollo y se concluye que los programas de cribado deben estar adaptados a cada país (17). En España no existe un programa de cribado común a todos los hospitales. Pallás sugiere que cada centro defina sus propios criterios de cribado en función de la experiencia adquirida y de los resultados obtenidos (16). Para Larsson las modificaciones de los criterios de cribado deben ser consideradas regularmente en cada hospital, pero exigen estudios prospectivos y consecutivos además de periódicas revisiones de la eficacia de los protocolos (18).

La tendencia al alza o a la baja de la incidencia de la ROP en los últimos años es también objeto de debate. La mayoría de autores señala un aumento en la incidencia y gravedad de la ROP en los RNPT más inmaduros y con muy bajo peso al nacer, coincidiendo con un incremento de la supervivencia de estos niños (3-5). Para otros autores, la excelencia en los cuidados neonatales, al margen del incremento de la supervivencia, puede ser la causa del aumento de la ROP grave entre los más inmaduros, porque la agresividad de los tratamientos e intervenciones necesarias para asegurar la supervivencia, puede iniciar la ROP (6). Entre estos tratamientos estaría el uso de surfactante pulmonar exógeno. Se han publicado resultados contradictorios en relación al uso de surfactante y la incidencia de ROP. Hussain señala una disminución de la incidencia y gravedad de la ROP (19), pero estudios posteriores han aclarado que si el surfactante se emplea como tratamiento del distrés respiratorio, la incidencia no varía o aumenta (20). En cambio, si se emplea como profilaxis, antes de que aparezca el distrés respiratorio, entonces sí disminuye la incidencia y gravedad de la ROP (21). La administración de eritropoyetina (rhEPO), que disminuye la necesidad de transfusiones, se relaciona con una más alta incidencia y gravedad de la ROP (22). También las transfusiones ejercen un efecto negativo en el desarrollo de la ROP (23). En España, Montañez señala un aumento en la incidencia de ROP en los últimos años (13), mientras Grunauer apunta un descenso (14). En Murcia desconocemos esta tendencia por no disponer de datos epidemiológicos previos y las limitaciones de nuestro estudio, que solo recoge la incidencia de un año. Son necesarios estudios pros- pectivos y consecutivos de varios años para aclarar esta cuestión.

En la región de Murcia un tercio de los prematuros cribados para ROP en el año 2004, según el protocolo actual, desarrolla la enfermedad y el $50 \%$ de ellos necesita tratamiento. Los resultados obtenidos en el presente estudio indican que los criterios de selección para cribado ROP actuales, $\leq 32$ semanas $\mathrm{EG}$ o $\leq 1.500 \mathrm{~g} \mathrm{PN}$, son adecuados para nuestra población y que con criterios más restrictivos se podría omitir el diagnóstico de ROP grave en algunos pacientes. Para modificar este protocolo precisamos más estudios.

\section{BIBLIOGRAFÍA}

1. Sola A, Chow L, Rogido M. Retinopathy of prematurity and oxygen therapy: a changing relationship. An Pediatr (Barc) 2005; 62: 48-63.

2. Palmer EA, Flynn JT, Hardy RJ, Phelps DL, Phillips CL, Schaffer DB, et al. Incidence and early course of retinopathy of prematurity. The Cryotherapy for Retinopathy of Prematurity Cooperative Group. Ophthalmology 1991; 98: 1628-1640.

3. Good WV, Hardy RJ, Dobson V, Palmer EA, Phelps DL, Quintos $M$, et al. The incidence and course of retinopathy of prematurity: findings from the early treatment for retinopathy of prematurity study. Pediatrics 2005; 116: 15 23.

4. Larsson E, Carle-Petrelius B, Cernerud G, Ots L, Wallin A, Holmstrom $G$. Incidence of ROP in two consecutive Swedish population based studies. Br J Ophthalmol 2002; 86: 1122-1126.

5. Hameed B, Shyamanur K, Kotecha S, Manktelow BN, Woodruff $G$, Draper ES, et al. Trends in the incidence of severe retinopathy of prematurity in a geographically defined population over a 10-year period. Pediatrics 2004; 113: 1653-1657.

6. Vyas J, Field D, Draper ES, Woodruff G, Fielder AR, Thompson J, et al. Severe retinopathy of prematurity and its association with different rates of survival in infants of less than 1,251 g birth weight. Arch Dis Child Fetal Neonatal Ed 2000; 82: F145-F149.

7. American Academy of Pediatrics. Section on ophthalmology. Screening examination of premature infants for retinopathy of prematurity. Pediatrics 2001; 108: 809-811.

8. Reynolds JD, Dobson V, Quinn GE, Fielder AR, Palmer EA, Saunders RA, et al. Evidence-based screening criteria for retinopathy of prematurity: natural history data from the CRYO-ROP and LIGHT-ROP studies. Arch Ophthalmol 2002; 120: 1470-1476.

9. An international classification of retinophaty of prematurity. The International Committee for the Classification of Retinopathy of Prematurity. Arch Ophthalmol 1984; 102: 1130-1134.

10. An international classification of retinophaty of prematurity: II. The classification of retinal detachment. The Inter- 
national Committee for the Classification on the Late Stages of Retinopathy of Prematurity. Arch Ophthalmol 1987; 105: 906-912.

11. Multicenter trial of cryotherapy for retinopathy of prematurity: preliminary results. Cryotherapy for Retinophaty of Prematurity Cooperative Group. Pediatrics 1988; 81: 697-706.

12. Early Treatment For Retinopathy Of Prematurity Cooperative Group. Revised indications for the treatment of retinopathy of prematurity: results of the early treatment for retinopathy of prematurity randomized trial. Arch Ophthalmol 2003; 121: 1684-1694.

13. Montañez, FJ, Olea JL. Once años de experiencia en el manejo de la retinopatía del prematuro en las Islas Baleares. Arch Soc Esp Oftalmol 2005; 80: 713-718.

14. Grunauer N, Iriondo Sanz, M, Serra Castanera A, Krauel Vidal J, Jiménez González R. Retinopatía del prematuro. Casuística de los años 1995-2001. An Pediatr (Barc) 2003; 58: 471-477.

15. Martín Begué N, Perapoch López J. Retinopatía del prematuro: incidencia, gravedad y evolución. An Pediatr (Barc) 2003; 58: 156-161.

16. Pallás Alonso C, de La Cruz Bertolo J, Tejada Palacios P, Fernández, $C$, Muñoz Labián $M$, Carreño Guerra $M$. Impacto de los criterios de cribado para la retinopatía de la prematuridad. Un año de experiencia. An Esp Pediatr 2001; 55: 53-57.

17. Gilbert C, Fielder A, Gordillo L, Quinn G, Semiglia R, Visintin $P$, et al. Characteristics of infants with severe retinopathy of prematurity in countries with low, moderate, and high levels of development: implications for screening programs. Pediatrics 2005; 115: e518-e525.

18. Larsson E, Holmstrom G. Screening for retinopathy of prematurity: evaluation and modification of guidelines. $\mathrm{Br}$ J Ophthalmol 2002; 86: 1399-1402.

19. Hussain N, Clive J, Bhandari V. Current incidence of retinopathy of prematurity, 1989-1997. Pediatrics 1999; 104: e26.

20. Kim TI, Sohn J, Pi SY, Yoon YH. Postnatal risk factors of retinopathy of prematurity. Paediatr Perinat Epidemiol 2004; 18: 130-134.

21. Termote J, Schalij-Delfos NE, Brouwers HA, Donders AR, Cats BP. New developments in neonatology: less severe retinopathy of prematurity? J Pediatr Ophthalmol Strabismus 2000; 37: 142-148.

22. Bierer R, Peceny MC, Hartenberger CH, Ohls RK. Erythropoietin concentrations and neurodevelopmental outcome in preterm infants. Pediatrics 2006; 118: e635-e640.

23. Seiberth $V$, Linderkamp $O$. Risk factors in retinopathy of prematurity. A multivariate statistical analysis. Ophthalmologica 2000; 214: 131-135. 